\begin{tabular}{llllll} 
O P E R A T I O N S R E S E A R C H A N D D E C I S I O N S \\
\hline No. 1
\end{tabular}

DOI: $10.37190 /$ ord 200102

\title{
A FUZZY APPROACH TO MULTI-OBJECTIVE MIXED-INTEGER LINEAR PROGRAMMING MODEL FOR MULTI-ECHELON CLOSED-LOOP SUPPLY CHAIN WITH MULTI-PRODUCT MULTI-TIME PERIOD
}

\author{
SEMA AKIN BAS ${ }^{1 *}$, BEYZA AHLATCIOGLU OZKOK $^{2}$ \\ ${ }^{1}$ Department of Mathematics, Faculty of Arts and Science, \\ Yildiz Technical University, Istanbul, Turkey \\ ${ }^{2}$ Department of Business Administration, Faculty of Economics \\ and Administrative Sciences, Yildiz Technical University, Istanbul, Turkey
}

\begin{abstract}
By the green point of view, supply chain management (SCM), which contains supplier and location selection, production, distribution, and inventory decisions, is an important subject being examined in recent years by both practitioners and academicians. In this paper, the closed-loop supply chain (CLSC) network that can be mutually agreed by meeting at the level of common satisfaction of conflicting objectives is designed. We construct a multi-objective mixed-integer linear programming (MOMILP) model that allows decision-makers to more effectively manage firms' closed-loop green supply chain (SC). An ecological perspective is brought by carrying out the recycling, remanufacturing and destruction to SCM in our proposed model. Maximize the rating of the regions in which they are located, minimize total cost and carbon footprint are considered as the objectives of the model. By constructing our model, the focus of customer satisfaction is met, as well as the production, location of facilities and order allocation are decided, and we also carry out the inventory control of warehouses. In our multi-product multi-component multi-time-period model, the solution is obtained with a fuzzy approach by using the min operator of Zimmermann. To illustrate the model, we provide a practical case study, and an optimal result containing a preferable level of satisfaction to the decision-maker is obtained.
\end{abstract}

Keywords: closed-loop supply chain management, multi-objective optimization, fuzzy mixed-integer linear programming, inventory decision

${ }^{*}$ Corresponding author, email address akins@yildiz.edu.tr Received 20 March 2019, revised 20 April 2020 


\section{Introduction}

The concept of the supply chain (SC) is the set of relationships and links that ensure the movement of products between suppliers, manufacturers, distributors, retailers, and ultimately consumers. Businesses have changed their perspective in the supply chain management (SCM) with rapidly growing environmental awareness and legislation. With the change of viewpoints of companies, the green image has not only helped to maintain ecological balance but also increased profitability by providing a competitive advantage. Ilgin and Gupta mentioned the evolution of environmentally conscious manufacturing and product recovery that has occurred in the last decade and considered the new areas that have come into focus during this time [1]. Recycling, which is one of the activities of greening the $\mathrm{SC}$, is a crucial process to prevent wasting of our resources that are getting more and more consumption day by day. Recycling, remanufacturing of used products and bringing them back to the market provide the environmental benefits and they also reduce production cost. The closed-loop supply chain (CLSC) can be thought of as an integrated form of traditional forward and environmentally sensitive reverse logistics activities. A general forward SC network consists of suppliers, manufacturers, distributors, and customers [2]. In reverse SC network, used products from customers are accumulated in collection centres and shipped them to refurbishing, disassembly, and decomposition centres.

Inventory decision is one of the critical decision classes because it is directly related to customer satisfaction. Furthermore, it enables to minimize the damage which may be occurred as a result of unexpected and unforeseen changes in customer demand and uncertainty in supply by determining the levels of raw materials and products be stocked in which facilities.

Fuzzy logic can be used in SC modelling or solving to deal with many real-life uncertainties such as demand uncertainty. The fuzzy set theory enables the decision-maker not only to consider all available options under given constraints (optimization) but also to develop new alternatives at the same time. Moreover, another vital issue that cannot be overlooked for firms in SCM is the ability to make multi-objective decisions. It is increasingly difficult to manage the SC for a single objective since the world is estimated hardly as a sole criterion and interpreted as if it were bound to an only measure.

In this paper, a multi-echelon multi-objective closed-loop supply chain (MOCLSC) model is proposed to periodically make decisions of supplier selection, production, facilities (e.g., warehouse, distribution and recycling centres) set-up, the transportation quantities, and the level of product(s) stock in a warehouse. The presented network is modelled as a mixed-integer linear programming (MILP) problem by paying regard to the economic and environmental factors. Our three model objectives are minimization of both the total cost and $\mathrm{CO}_{2}$ emission, which is a greenhouse gas, and maximizing the 
percentage of importance previously attributed to the facilities. We have solved the linear programming (LP) problem by using the minimum operator of Zimmermann with a fuzzy approach to maximize the mutual satisfaction of these all objectives. The proposed model is dealt with using GAMS 23.5.

The rest of the paper is arranged as follows: Related works done by the previous researchers are briefly reviewed in the next section. The proposed CLSC framework and the mathematical model are mentioned in Section 3. Section 4 describes the solution methodology and there is a case study to test the model in Section 5. Finally, Section 6 represents general conclusions.

\section{Literature review}

Under the influence of the Industrial Revolution, Bowersox introduced to the bases of the SC, so-called physical distribution phase, in the period from the First and Second World Wars to the 1960s [3]. Since the day it comes, there are many studies on SC which has attracted considerable attention from companies and researchers. Tsiakis et al. [4] considered the design of multi-product multi-echelon SC networks which is modelled mathematically as a MILP optimization problem. Melo et al. [5] gave a literature review of facility location models in the context of SCM. Mousavi et al. [6] considered the design of a two-echelon distribution SC network for the seasonal products with multiple vendors (manufacturers), buyers (retailers) and a set of warehouses for each vendor.

With globalization and increasing environmental sensitivity, scientists turn towards green CLSC issues. Hu et al. [7] presented a cost-minimization discrete-time linear analytical model for a multi-time-step, multi-type hazardous-waste reverse logistics system. Savaskan et al. [8] handled the problem of choosing the appropriate reverse channel structure for the collection of used products from customers. Kim et al. [9] proposed a general framework for remanufacturing and studied on a mathematical model to maximize the total cost savings by optimally deciding the number of parts to be processed at each remanufacturing facility, the number of purchased parts from the subcontractor. Abdallah et al. [10] introduced the uncapacitated closed-loop location inventory model, which captures the interdependency between location inventory decisions in the forward and reverse SCs. Ozceylan and Paksoy [11] proposed a new mixed-integer mathematical model for a CLSC network that includes both forward and reverse flows with multiperiods and multi-parts with minimizing the objective function. Hasanov et al. [12] addressed the coordination of order quantities in a four-level closed-loop supply chain (CLSC) with remanufacturing.

In the modelling and solving of CLSC problems, it has been concentrated on studies involving fuzzy mathematics due to insufficient crisp approaches and uncertainties concerning the nature of life. Chen and Lee [13] proposed a multi-product, multi-stage, and 
multi-period scheduling model to deal with multiple incommensurable goals for a multiechelon SC network with uncertain market demands and product price. Peidro et al. [14] proposed a fuzzy mathematical programming model for SC planning which considers supply, demand, and process uncertainties. The model is formulated as a fuzzy MILP model where data is ill-known and identified by triangular fuzzy numbers. Ozkok and Tiryaki [15] proposed a compensatory fuzzy approach to solve multi-objective linear supplier selection problem with multiple-item by using Werners' "fuzzy and" $\left(\mu_{\text {and }}\right)$ operator. Shi et al. [16] studied the production planning problem for a multi-product closed-loop system and aimed to maximize the manufacturer's expected profit by jointly determining the production quantities of brand-new products, the amounts of remanufactured products, and the acquisition prices of the used products. Amin and Zhang [17] developed an integrated model that has a framework for supplier selection criteria in reverse logistics with a fuzzy method on qualitative criteria and they proposed a MOMILP model to take strategic and tactical decisions. Pishvaee and Razmi [2] proposed a multi-objective fuzzy mathematical programming model for designing an environmental SC under the inherent uncertainty of input data in such a problem. Ramezani et al. [18] addressed the application of fuzzy sets to design a multi-product, multi-period CLSC network. Jindal and Sangwan [19] proposed a multi-product, multi-facility capacitated CLSC framework in an uncertain environment including reuse, refurbish, recycle, and disposal of parts. Jindal et al. [20] proposed the network design and optimization of a multiproduct, multi-time, multi-echelon capacitated CLSC in an uncertain environment. Soleimani et al. [21] carried out by accounting for environmental considerations, total profit optimization, and reduction of lost working days due to occupational accidents, as well as maximizing responsiveness to customer demand. Chen et al. [22] established a model which structured and optimized the process of product refurbishment, considering inventories, and uncertainties. Wu et al. [23] designed a fuzzy mixed-integer linear programming model for a closed-loop supply chain network design under uncertainty and constructed a two-stage interactive possibilistic programming procedure.

As can be understood from the literature review, many factors which are effective in SC designs can be extended to more real-life problems by expanding networks. Therefore, we aimed to gain the literature by building a model for MOCLSC management, where optimal distribution can be achieved. In the model, it is decided procurement raw materials, manufacture in the plants, and usage of facilities (warehouses, distribution centres, recycling centres) periodically. Besides, stock control in the warehouse(s) is a crucial decision for our model to be released in case of uncertainties such as raw material inefficiency. In other words, it is performed optimal location and allocation to different plants, warehouses, distribution centres, recycling centres, and external suppliers in the model. Considering the increasing environmental sensitivity in recent years, it is aimed to reduce $\mathrm{CO}_{2}$ emission during operations such as production, handling, transportation, recycling, destruction. The model also takes into consideration to give priority to the use of the facilities, which will benefit the companies. 


\section{Multi-objective closed-loop supply chain framework and the mathematical model}

\subsection{The proposed CLSC framework}

As illustrated in Fig. 1, the CLSC framework is presented for handling the multi-echelon network in which forward flow, reverse flow, and their mutual interactions are considered simultaneously. The network is structured multi-time model with considering the inventory level of products and the related inventory costs.

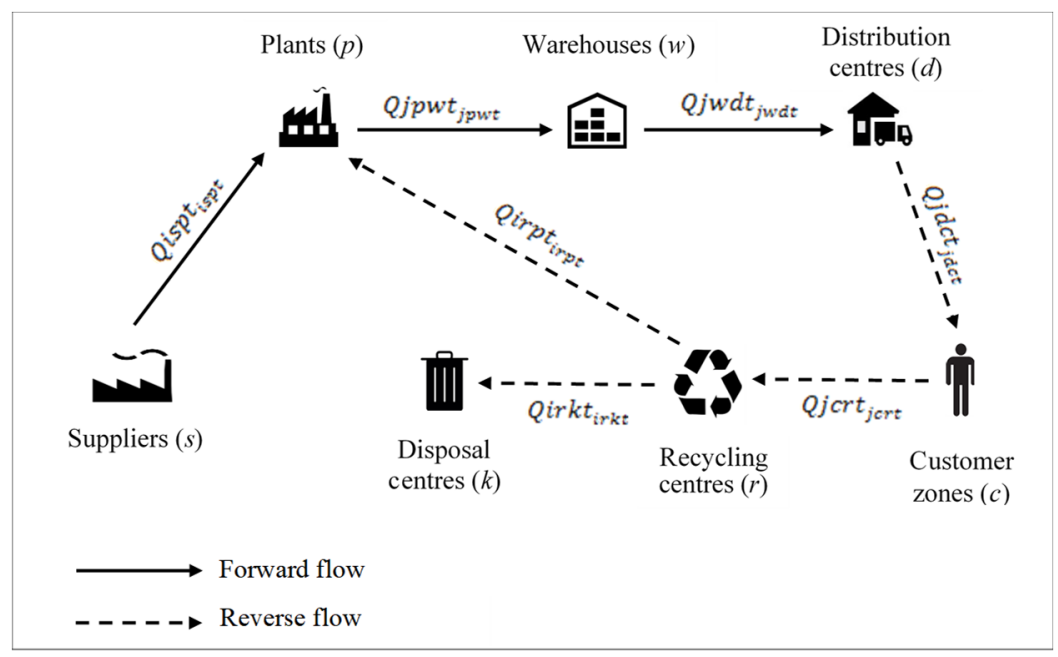

Fig. 1. The proposed CLSC framework

Forward SC is consisting of suppliers, plants, warehouses which is holding inventory levels of products, distribution centres, and customer zones. The reverse SC starts with the collection of returned products from the customer zones to recycling centres. The network will be managed as efficiently as possible by managing the inventory of products, which is a critical decision in the time-period that has products with high production and logistical costs. Since a product consists of various components/parts, the returned products are disassembled at recycling centres for remanufacturing and disposal of the components/parts processes. The mathematical model created in the article is based on the assumptions as follows:

- All objectives and constraints are linear.

- The locations of the suppliers, the customer zones, and the disposal centres are fixed and known in advance.

- A product consists of various components/parts. 
- The distances between the facilities are determined and fixed throughout the planning periods.

- The unit transportation costs calculated based on distances and the amount of unit $\mathrm{CO}_{2}$ emission due to these transports are determined.

- Quantity discounts are not taken into consideration.

- Inventory is stored at the warehouses. Inventory level is managed throughout the network, and storage conditions of the warehouses are available for product types.

- Each plant, warehouse, distribution centre, and recycling centre has rating values that indicate a predetermined level of preference.

- The decision is taken periodically, and each period has a link to the previous period.

- The recycled components/parts have the same quality as the brand-new parts and can be used manufacturing process at the plants.

\subsection{Model development}

The proposed multi-objective multi-period multi-product mixed-integer linear programming (MILP) model is expressed in this section. The set of indices, decision variables, parameters, constraints, and objectives of the MILP model to be used in the case study are as follows:

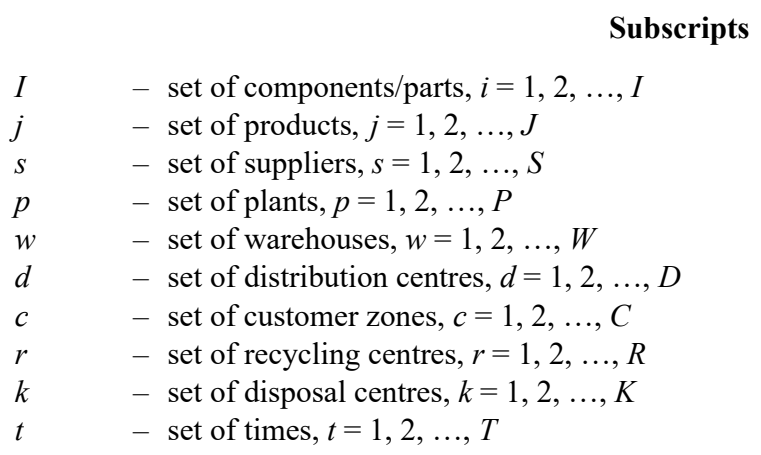

\section{Decision variables}

Yjpt $_{j p t}$ - binary variable for the usage of plant $p$ for product $j$ in time $t$

$Y_{j w t_{j w t}}$ - binary variable for the usage of warehouse $w$ for product $j$ in time $t$

$Y j d t_{j d t}$ - binary variable for the usage of distribution centre $d$ for product $j$ in time $t$

Yjrt $_{j r t}$ - binary variable for the usage of recycling centre $r$ for product $j$ in time $t$

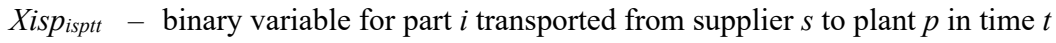

$X j p w t_{j p w t}$ - binary variable for product $j$ transported from plant $p$ to warehouse $w$ in time $t$

$X j w d t_{j w d t}$ - binary variable for product $j$ transported from warehouse $w$ to distribution centre $d$ in time $t$

$X j d c t_{j d c t}$ - binary variable for product $j$ transported from distribution centre $d$ to customer zone $c$ in time $t$

$X j c r t_{j c r t}-$ binary variable for product $j$ transported from customer zone $c$ to recycling centre $r$ in time $t$

Xirptirpt - binary variable for part $i$ transported from recycling centre $r$ to plant $p$ in time $t$ 
Xirktirkt - binary variable for part $i$ transported from recycling centre $r$ to disposal centre $k$ in time $t$

$L S j w t_{j w t}$ - inventory level of product $j$ in warehouse $w$ in time $t$

Qjptjpt - units of product $j$ to be produced in plant $p$ in time $t$

Qisptispt - units of part $i$ to be purchased from supplier $s$ to plant $p$ in time $t$

Qjpwt $t_{j p w}$ - units of product $j$ to be transported from plant $p$ to warehouse $w$ in time $t$

Qjwd $t_{j w d t}$ - units of product $j$ to be obtained in distribution centre $d$ from warehouse $w$ in time $t$

Qjdct $_{j d c t}$ - units of product $j$ to be transported from distribution centre $d$ to customer zone $c$ in time $t$

Qjcrtjcrt - units of product $j$ to be recycled in recycling centre $r$ from customer zone $c$ in time $t$

Qirptirpt - units of part $i$ to be transported from recycling centre $r$ to plant $p$ in time $t$

Qirktirkt - units of part $i$ to be destroyed in disposal centre $k$ from recycling centre $r$ in time $t$

\section{Parameters}

$D j c t_{j c t}-$ demand of customer zone $c$ for product $j$ in time $t$

$c i j_{i j} \quad-$ units of part $i$ in product $j$

$S C j p_{j p} \quad-$ usage cost for plant $p$ of product $j$

$S C j w_{j w} \quad$ - usage cost for warehouse $w$ of product $j$

$S C j d_{j d} \quad-$ usage cost for distribution centre $d$ of product $j$

$S C j r_{j r} \quad$ - usage cost for recycling centre $r$ of product $j$

$P C i s p_{i s p} \quad-$ unit purchasing cost for part $i$ from supplier $s$ to plant $p$

$P C j p t_{j p t}-$ unit cost of production for product $j$ in plant $p$ in time $t$

$S t C j w t_{j w t}$ - unit cost of inventory holding for product $j$ in warehouse $w$ in time $t$

$R C j r_{j r} \quad-$ unit cost of recycling for product $j$ in recycling centre $r$

$D C i k_{i k} \quad-$ unit cost of disposal for part $i$ in disposal centre $k$

TCisp $_{i s p} \quad$ - unit transportation cost for part $i$ transported from supplier $s$ to plant $p$

$T C j p w_{j p w}-$ unit transportation cost for product $j$ transported from plant $p$ to warehouse $w$

$T C j w d_{j w d}-$ unit transportation cost for product $j$ transported from warehouse $w$ to distribution centre $d$

$T C j d c_{j d c}$ - unit transportation cost for product $j$ transported from distribution centre $d$ to customer zone $c$

TCjcr $_{j c r} \quad-$ unit transportation cost for product $j$ transported from customer zone $c$ to recycling centre $r$

TCirp $_{\text {irp }} \quad-$ unit transportation cost for part $i$ transported from recycling centre $r$ to plant $p$

TCirk $_{i r k} \quad$ - unit transportation cost for part $i$ transported from recycling centre $r$ to disposal centre $k$

Capjpt $_{j p t}-$ production capacity of product $j$ at plant $p$ in time $t$

Capjwt $_{j w t}$ - capacity of warehouse $w$ for product $j$ in time $t$

Capjdt $_{j d t}$ - capacity of distribution centre $d$ for product $j$ in time $t$

Capjrt $_{j r t}$ - capacity of recycling centre $r$ for product $j$ in time $t$

Capikt $_{i k t}$ - capacity of disposal centre $k$ for part $i$ in time $t$

$L B i \quad-$ lower bound of part quantity per transportation

$L B j \quad$ - lower bound of product quantity per transportation

MXis $_{i s} \quad$ - maximum purchase order from supplier $s$ in time $t$ 


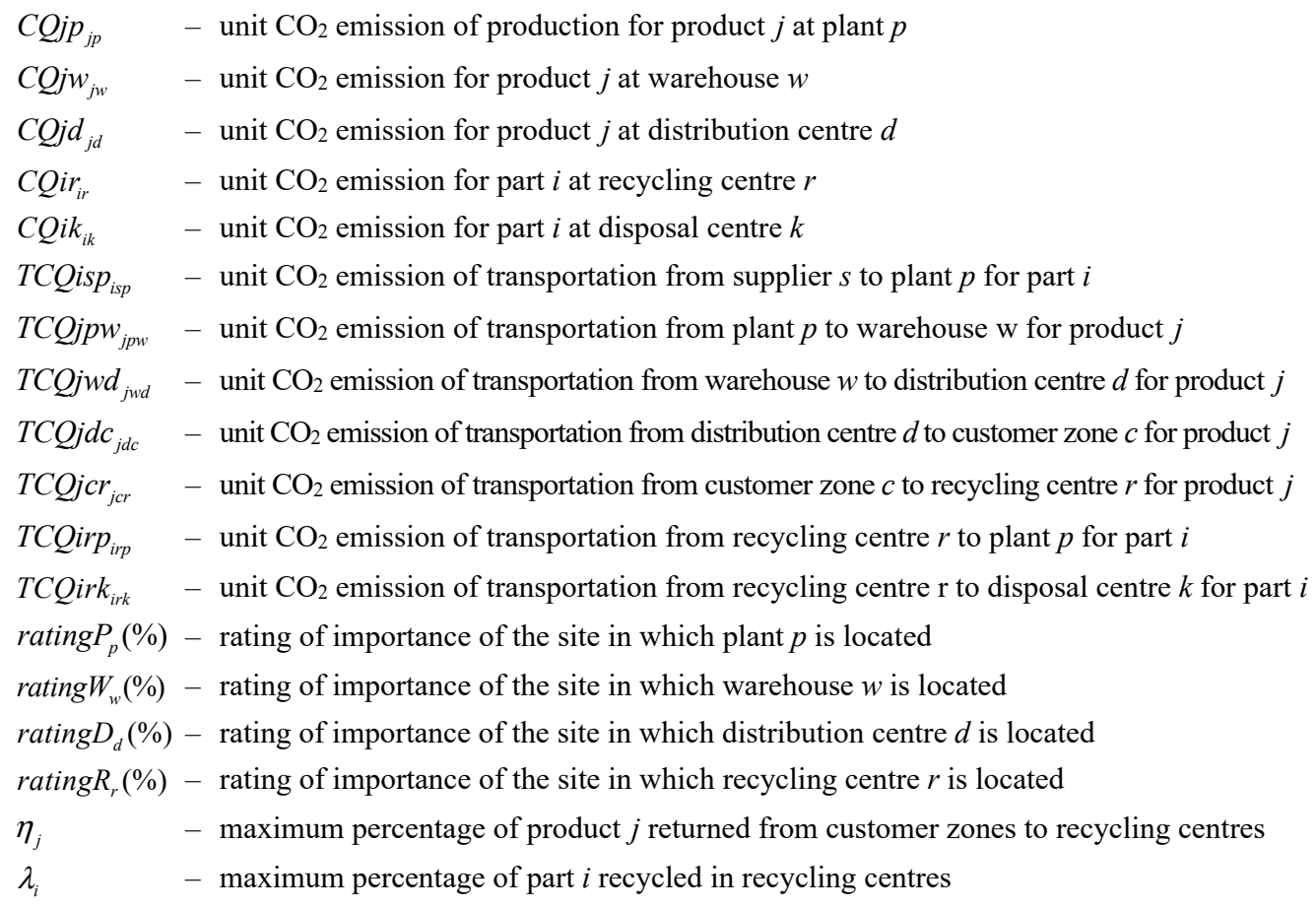

\section{Objective functions}

The objectives are modelled as three functions: (i) minimization of total cost, (ii) maximization of rating of importance, and (iii) minimization of total $\mathrm{CO}_{2}$ emission.

To minimize the total cost, the following costs are considered: purchasing components from suppliers, manufacturing, holding inventory, recycling, disposal, transportation, and usage of facilities.

$$
\begin{aligned}
& \min Z_{1}=\sum_{i}^{I} \sum_{s}^{S} \sum_{p}^{P} \text { TCisp }_{i s p} \sum_{t}^{T} \text { Qispt }_{\text {ispt }}+\sum_{j}^{J} \sum_{p}^{P} \sum_{w}^{W} T C j p w_{j p w} \sum_{t}^{T} \text { Qjpwt }_{j p w t} \\
& +\sum_{j}^{J} \sum_{w}^{W} \sum_{d}^{D} T C j w d_{j w d} \sum_{t}^{T} Q j w d t_{j w d t}+\sum_{j}^{J} \sum_{d}^{D} \sum_{c}^{C} T C j d c_{j d c} \sum_{t}^{T} Q j d c t_{j d c t} \\
& +\sum_{j}^{J} \sum_{c}^{C} \sum_{r}^{R} \text { TCjcr }_{j c r} \sum_{t}^{T} \text { Qjert }_{j c r t}+\sum_{i}^{I} \sum_{r}^{R} \sum_{p}^{P} \text { TCirp }_{i r p} \sum_{t}^{T} \operatorname{Qirpt}_{i r p t} \\
& +\sum_{i}^{I} \sum_{r}^{R} \sum_{k}^{K} \text { TCirk }_{i r k} \sum_{t}^{T} \text { Qirkt }_{i r k t}+\sum_{j}^{J} \sum_{p}^{P} \sum_{t}^{T} P^{T} C_{j p t_{j p t}} \text { Qjpt }_{j p t} \\
& +\sum_{j}^{J} \sum_{r}^{R} R C j r_{j r} \sum_{c}^{C} \sum_{t}^{T} Q_{j c r t}{ }_{j c r t}+\sum_{i}^{I} \sum_{k}^{K} D C i k_{i k} \sum_{r}^{R} \sum_{t}^{T} \operatorname{Qirkt}_{i r k t}
\end{aligned}
$$




$$
\begin{aligned}
& +\sum_{j}^{J} \sum_{p}^{P} S C j p_{j p} \sum_{t}^{T} Y j p t_{j p t}+\sum_{j}^{J} \sum_{w}^{W} S C j w_{j w} \sum_{t}^{T} Y j w t_{j w t} \\
& +\sum_{j}^{J} \sum_{d}^{D} S C j d_{j d} \sum_{t}^{T} Y j d t_{j d t}+\sum_{j}^{J} \sum_{r}^{R} S C j r_{j r} \sum_{t}^{T} Y j r t_{j r t} \\
& +\sum_{j}^{J} \sum_{w}^{W} \sum_{t}^{T} L S j w t_{j w t} . S t C j w t_{j w t}+\sum_{i}^{I} \sum_{s}^{S} \sum_{p}^{P} P C i s p_{i s p} \sum_{t}^{T} \text { Qispt }_{i s p t}
\end{aligned}
$$

The aim of the maximization of the rating of importance is to decide on the way to maximize the important values that indicate the preference level of the candidate facilities.

$$
\begin{aligned}
\max Z_{2}= & \sum_{p}^{P} \operatorname{rating} P_{p} \sum_{j}^{J} \sum_{t}^{T} Q j p t_{j p t}+\sum_{w}^{W} \operatorname{rating} W_{w} \sum_{j}^{J} \sum_{p}^{P} \sum_{t}^{T} Q j p w t_{j p w t} \\
& +\sum_{d}^{D} \operatorname{rating} D_{d} \sum_{j}^{J} \sum_{w}^{W} \sum_{t}^{T} Q j w d t_{j w d t}+\sum_{r}^{R} \operatorname{rating} R_{r} \sum_{j}^{J} \sum_{c}^{C} \sum_{t}^{T} Q j c r t_{j c r t}
\end{aligned}
$$

The aim of the minimization of the total $\mathrm{CO}_{2}$ emission is to reduce the carbon footprint to the minimum, taking into account the emissions during processing at facilities and transportation.

$$
\begin{aligned}
& \min Z_{3}=\sum_{i}^{I} \sum_{s}^{S} \sum_{p}^{P} \text { TCQisp }_{i s p} \sum_{t}^{T} \text { Qispt }_{\text {ispt }}+\sum_{j}^{J} \sum_{p}^{P} \sum_{w}^{W} \text { TCQjpw }_{j p w} \sum_{t}^{T} \text { Qjpwt }_{j p w t} \\
& +\sum_{j}^{J} \sum_{w}^{W} \sum_{d}^{D} T C Q j w d_{j w d} \sum_{t}^{T} Q j w d t_{j w d t}+\sum_{j}^{J} \sum_{d}^{D} \sum_{c}^{C} T C Q j d c_{j d c} \sum_{t}^{T} Q_{j} d c t_{j d c t} \\
& +\sum_{j}^{J} \sum_{c}^{C} \sum_{r}^{R} \text { TCQjer }_{j c r} \sum_{t}^{T} \text { Qjcrt }_{j c r t}+\sum_{i}^{I} \sum_{r}^{R} \sum_{p}^{P} \text { TCQirp }_{i r p} \sum_{t}^{T} \text { Qirpt }_{\text {irpt }} \\
& +\sum_{i}^{I} \sum_{r}^{R} \sum_{k}^{K} \text { TCQirk }_{i r k} \sum_{t}^{T} \text { Qirkt }_{\text {irkt }}+\sum_{j}^{J} \sum_{p}^{P} \text { CQjp }_{j p} \sum_{t}^{T} \text { Qjpt }_{j p t} \\
& +\sum_{j}^{J} \sum_{w}^{W} C Q j w_{j w} \sum_{t}^{T}\left(L S j w t_{j w, t-1}+\sum_{p}^{P} Q j p w t_{j p w t}\right) \\
& +\sum_{j}^{J} \sum_{d}^{D} C Q j d_{j d} \sum_{c}^{C} \sum_{t}^{T} \text { Qjdct }_{j d c t}+\sum_{i}^{I} \sum_{r}^{R} C \operatorname{Qir}_{i r} \sum_{p}^{P} \sum_{t}^{T} \text { Qirpt }_{\text {irpt }} \\
& +\sum_{i}^{I} \sum_{k}^{K} C Q i k_{i k} \sum_{r}^{R} \sum_{t}^{T} \text { Qirkt }_{i r k t}
\end{aligned}
$$




\section{Subject to constraints}

Constraint (4) ensures that the demand for each product is satisfied with the sum of newly produced products.

$$
D j c t_{j c t}=\sum_{d}^{D} Q j d c t_{j d c t} \quad \forall j, \forall c, \forall t
$$

Constraint (5) provides a balance of parts to be constituted products. Constraint (6) ensures that the amount of production in a plant is equal to the amount that goes to warehouses; thus, it hinders the inventory of products in the plant. (7) and (8) are balance constraints for distribution centres and recycling centres, while (9) and (10) are constraints for the recycling process and (11) for the destruction process.

$$
\begin{aligned}
& \sum_{s}^{S} \operatorname{Qispt}_{i s p t}+\sum_{r}^{R} \operatorname{Qirpt}_{i r p t}=\sum_{j}^{J} c i j_{i j} \text { Qjpt }_{j p t}, \quad \forall i, \forall p, \forall t \\
& Q j p t_{j p t}=\sum_{w}^{W} Q j p w t_{j p w t}, \quad \forall j, \forall p, \forall t \\
& \sum_{w}^{W} Q j w d t_{j w d t}=\sum_{c}^{C} Q j d c t_{j d c t}, \quad \forall j, \forall d, \forall t \\
& \sum_{j}^{J}\left(\sum_{c}^{C} \text { Qjcrt }_{j c r t}\right) c i j_{i j}=\sum_{p}^{P} \operatorname{Qirpt}_{i r p t}+\sum_{k}^{K} \operatorname{Qirkt}_{i r k t}, \quad \forall i, \forall r, \forall t \\
& \sum_{r}^{R} Q j c r t_{j c r t}=\eta_{j} \sum_{d}^{D} Q j d c t_{j d c t}, \quad \forall j, \forall c, \forall t \\
& \sum_{p}^{P} \operatorname{Qirpt}_{i r p t}=\lambda_{i} \sum_{j}^{J}\left(\sum_{c}^{C} \operatorname{Qjcrt}_{j c r t}\right) c i j_{i j}, \quad \forall i, \forall r, \forall t \\
& \sum_{k}^{K} \operatorname{Qirkt}_{i r k t}=\left(1-\lambda_{i}\right) \sum_{j}^{J}\left(\sum_{c}^{C} \operatorname{Qjcrt}_{j c r t}\right) c i j_{i j}, \quad \forall i, \forall r, \forall t
\end{aligned}
$$

Constraint (12) controls the inventory level of the product in the potential warehouse by providing to use of products, which stocked periodically, in the next period.

$$
\sum_{p}^{P} Q j p w t_{j p w t}+L S j w t_{j w, t-1}=\sum_{d}^{D} Q j w d t_{j w d t}+L S j w t_{j w t}, \quad \forall j, \forall w, \forall t
$$


While (13) is a production capacity constraint for plants, (14)-(18) are the capacity constraints for products entering and leaving warehouses, distribution centres, and recycling centres, respectively. Constraint (19) provides the upper limit for purchases from suppliers, and constraint (20) is the destruction limit for the potential disposal centres. If there is a connection between the supplier and the plant, Constraint (21) guarantees that the transport quantity is not exceeded by the maximum amount that can be purchased from the supplier. Constraints (22)-(27) provide that no transportation occurs if there is no connection between the facilities and the customer zones.

$$
\begin{aligned}
& \sum_{w}^{W} \text { Qjpwt }_{j p w t} \leq \operatorname{Capjpt}_{j p t} Y_{j p t_{j p t}}, \quad \forall j, \forall p, \forall t \\
& \sum_{p}^{P} \operatorname{Qjpwt}_{j p w t} \leq \operatorname{Capjwt}_{j w t} Y_{j w t_{j w t}}, \quad \forall j, \forall w, \forall t \\
& \sum_{d}^{D} Q j w d t_{j w d t} \leq \operatorname{Capjwt}_{j w t} Y_{j w t_{j w t}}, \quad \forall j, \forall w, \forall t
\end{aligned}
$$

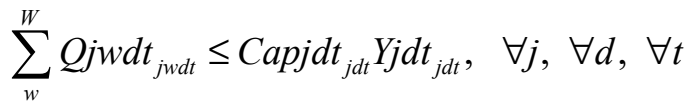

$$
\begin{aligned}
& \sum_{c}^{C} Q_{j d c t_{j d c t}} \leq C a p j d t_{j d t} Y j d t_{j d t}, \quad \forall j, \forall d, \forall t \\
& \sum_{c}^{C} \operatorname{Qjcrt}_{j c r t} \leq \operatorname{Capjrt}_{j r t} Y_{j r t} t_{j r t}, \quad \forall j, \forall r, \forall t \\
& \sum_{p}^{P} \operatorname{Qispt}_{i s p t} \leq M X i s_{i s}, \quad \forall i, \forall s, \forall t \\
& \sum_{r}^{R} \text { Qirkt }_{i r k t} \leq \text { Capikt }_{i k t}, \quad \forall i, \forall k, \forall t \\
& \operatorname{Qispt}_{i s p t} \leq M X i s_{i s} X_{i s p t} t_{i s p t}, \forall i, \forall j, \forall s, \forall p, \forall t \\
& \operatorname{Qjpwt}_{j p w t} \leq \operatorname{Capjpt}_{j p t} X_{j p w t_{j p w t}} \quad \forall j, \forall p, \forall w, \forall t
\end{aligned}
$$




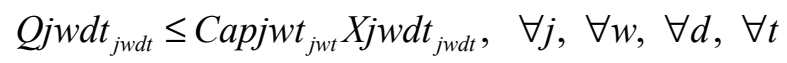

$$
\begin{aligned}
& Q_{j d c t_{j d c t}} \leq \operatorname{Capjdt}_{j d t} X_{j d c t} t_{j d c t}, \quad \forall j, \forall d, \forall c, \forall t \\
& \operatorname{Qjcrt}_{j c r t} \leq \operatorname{Capjrt}_{j r t} \text { Xjcrt }_{j c r t}, \quad \forall j, \forall c, \forall r, \forall t \\
& \operatorname{Qirpt}_{i r p t} \leq \operatorname{Capjrt}_{j r t} \text { Xirpt }_{i r p t}, \quad \forall i, \forall j, \forall r, \forall p, \forall t \\
& \text { Qirkt }_{i r k t} \leq \text { Capjkt }_{j k t} \text { Xirkt }_{i r k t}, \quad \forall i, \forall r, \forall k, \forall t
\end{aligned}
$$

Constraints (28)-(34) guarantee that the quantities to be transported do not fall below an accurate value of possible transportation. Constraints (35)-(38) allow at least one of the factories, warehouses, distribution centres, and recycling centres to be used, respectively. According to constraints (39)-(47), if the potential plant, warehouse, distribution centre, or recycling centre are not used, no connection is established with the facility in the same time. Constraints (48)-(56) allow at least one link to be found in addition to that when any plant, warehouse, distribution centre, or recycling centre receives a decision to use it.

$$
\begin{aligned}
& \text { Qispt }_{\text {ispt }} \geq L \text { LiXispt } t_{\text {ispt }}, \quad \forall i, \forall s, \forall p, \forall t \\
& Q_{j p w t_{j p w t}} \geq L B j X j p w t_{j p w t}, \quad \forall j, \forall p, \forall w, \forall t \\
& Q_{j w d t_{j w d t}} \geq L B j X j w d t_{j w d t}, \quad \forall j, \forall w, \forall d, \forall t \\
& Q_{j d c t_{j d c t}} \geq L B j X j d c t_{j d c t}, \quad \forall j, \forall d, \forall c, \forall t \\
& \operatorname{Qjcrt}_{j c r t} \geq L_{B j X j} \text { jcrt }_{j c r t}, \quad \forall j, \forall c, \forall r, \forall t \\
& \text { Qirpt }_{i r p t} \geq L_{\text {BiXirpt }}{ }_{i r p t}, \quad \forall i, \forall r, \forall p, \forall t \\
& \text { Qirkt }_{i r k t} \geq \text { LBiXirkt }_{i r k t}, \quad \forall i, \forall r, \forall k, \forall t \\
& \sum_{p}^{P} Y j p t_{j p t} \geq 1, \quad \forall j, \forall t
\end{aligned}
$$




$$
\begin{aligned}
& \sum_{w}^{W} Y j w t_{j w t} \geq 1, \quad \forall j, \forall t \\
& \sum_{d}^{D} Y_{j} d t_{j d t} \geq 1, \quad \forall j, \quad \forall t \\
& \sum_{r}^{R} \text { Yjrt }_{j r t} \geq 1, \quad \forall j, \quad \forall t \\
& X_{\text {ispt }} t_{i s p t} \leq \sum_{j}^{J} \text { Yjpt }_{j p t}, \quad \forall i, \forall s, \forall p, \forall t \\
& X j p w t_{j p w t} \leq Y j p t_{j p t}, \quad \forall j, \forall p, \forall w, \forall t \\
& X j p w t_{j p w t} \leq Y j w t_{j w t}, \quad \forall j, \forall p, \forall w, \forall t \\
& X j w d t_{j w d t} \leq Y j w t_{j w t}, \quad \forall j, \forall w, \forall d, \forall t \\
& X j w d t_{j w d t} \leq Y j d t_{j d t}, \quad \forall j, \forall w, \forall d, \forall t \\
& X j d c t_{j d c t} \leq Y j d t_{j d t}, \quad \forall j, \forall d, \forall c, \forall t \\
& X j c r t_{j c r t} \leq Y_{j r t} t_{j r t}, \quad \forall j, \forall c, \forall r, \forall t
\end{aligned}
$$

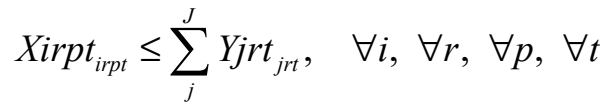

$$
\begin{aligned}
& X_{\text {irpt }} \text { irpt } \leq \sum_{j}^{J} \text { Yjpt }_{j p t}, \quad \forall i, \forall r, \forall p, \forall t \\
& \sum_{s}^{S} \sum_{i}^{I} X i s p t_{i s p t} \geq Y_{j p t_{j p t}}, \quad \forall j, \quad \forall p, \quad \forall t \\
& \sum_{w}^{W}{X j p w t_{j p w t} \geq Y_{j p t}}_{j p t}, \quad \forall j, \forall p, \forall t
\end{aligned}
$$




$$
\begin{aligned}
& \sum_{p}^{P} X j p w t_{j p w t} \geq Y j w t_{j w t}, \quad \forall j, \forall w, \forall t \\
& \sum_{d}^{D} X j w d t_{j w d t} \geq Y j w t_{j w t}, \quad \forall j, \forall w, \forall t \\
& \sum_{w}^{W} X j w d t_{j w d t} \geq Y j d t_{j d t}, \quad \forall j, \forall d, \forall t \\
& \sum_{c}^{C} X j d c t_{j d c t} \geq Y j d t_{j d t}, \quad \forall j, \forall d, \forall t \\
& \sum_{c}^{C} X j c r t_{j c r t} \geq Y_{j r t}{ }_{j r t}, \quad \forall j, \forall r, \forall t \\
& \sum_{p}^{P} \sum_{i}^{I}{X i r p t_{i r p t} \geq Y_{j r t}}_{j p t}, \quad \forall j, \forall r, \forall t \\
& \sum_{r}^{R} \sum_{i}^{I} X i r p t_{i r p t} \geq Y j p t_{j p t}, \quad \forall j, \forall p, \forall t
\end{aligned}
$$

The following constraints are related to binary and general integer values of the decision variables. All the decision variables are positive numbers.

$$
\begin{aligned}
& Y_{j p t_{j p t}},{Y j w t_{j w t}}, Y_{j d t} t_{j t}, Y_{j r t} t_{j r t} \in[0,1] \\
& X i s p t_{i s p t}, X j p w t_{j p w t}, X j w d t_{j w d t}, X j d c t_{j d c t}, X j c r t_{j c r t}, X i r p t_{i r p t}, X i r k t_{i r k t} \in[0,1] \\
& L S j w t_{j w t},{Q j p t_{j p t}}, Q_{i s p t} t_{i s p t}, Q j p w t_{j p w t}, \\
& Q j w d t_{j w d t}, Q_{j d c t}{ }_{j d c t}, Q_{j c r t}{ }_{j c r t}, Q_{i r p t} t_{i r p t}, Q_{i r k t}{ }_{i r k t} \geq 0
\end{aligned}
$$

\section{Fuzzy approach to the solution}

The decision processes with different relative values are based on a single purpose such as profit maximization, cost minimization, minimization of the working time. 
Gaining meaningful information by considering the degree of satisfaction of the objective for different decision options is the first of two main issues that are prioritized in multi-objective decision making. The other is to rank or weight the relative importance value for each. From this point of view, the initial process for the proposed solution approach of the multi-objective linear programming (MOLP) problem given by mathematically crisp data is to establish the membership functions of the objectives.

To determine the membership functions, the upper $\left(z_{k}^{+}\right)$and lower $\left(z_{k}^{-}\right)$bounds corresponding to respectively the best and worst satisfaction levels of the objective functions $z_{k}$, $k=1,2, \ldots, K$, should be determined. Therefore, each objective is minimized and maximized under the original constraints of the problem. Let $S$ be the feasible solutions region of the problem with the original constraints. Limit values are $z_{k}^{-}=\min _{x \in S} z_{k}(x)$, $z_{k}^{+}=\max _{x \in S} z_{k}(x)$. Membership functions should be defined after the boundary values are obtained. We use a linear membership function for the sake of simplicity. The linear membership function $\mu_{k}\left(z_{k}(x)\right)$ corresponding to the $k$ th objective function for maximization can be characterized as follows:

$$
\mu_{k}\left(z_{k}(x)\right)=\left\{\begin{array}{l}
0, \quad z_{k}(x)<z_{k}^{-} \\
\frac{z_{k}(x)-z_{k}^{-}}{z_{k}^{+}-z_{k}^{-}}, \quad z_{k}^{-} \leq z_{k}(x) \leq z_{k}^{+} \\
1, \quad z_{k}(x)>z_{k}^{+}
\end{array}\right.
$$

where $z_{k}^{-} \neq z_{k}^{+}, k=1,2, \ldots, n$; in the event that $z_{k}^{-}=z_{k}^{+}, \mu_{k}\left(z_{k}(x)\right)=1$. With similar logic, the linear membership function $\mu_{k}\left(z_{k}(x)\right)$ corresponding to the $k$ th objective function for minimization can be adapted as follows:

$$
\mu_{k}\left(z_{k}(x)\right)=\left\{\begin{array}{l}
1, \quad z_{k}(x)<z_{k}^{-} \\
\frac{z_{k}^{+}-z_{k}(x)}{z_{k}^{+}-z_{k}^{-}}, \quad z_{k}^{-} \leq z_{k}(x) \leq z_{k}^{+} \\
0, \quad z_{k}(x)>z_{k}^{+}
\end{array}\right.
$$

where $z_{k}^{-} \neq z_{k}^{+}, k=n+1, n+2, \ldots, K$; in the event that $z_{k}^{-}=z_{k}^{+}, \mu_{k}\left(z_{k}(x)\right)=1$ [14].

Using the minimum operator of Zimmermann [24], the updated form of the MOLPP is as follows: 


$$
\begin{aligned}
& \max _{x} \min _{k} \mu_{k}\left(z_{k}(x)\right) \\
& \text { s.t. } \\
& x \in S
\end{aligned}
$$

Using an auxiliary $\lambda$ variable (namely $\min _{k} \mu_{k}\left(z_{k}(x)\right)=\lambda \Rightarrow \mu_{k}\left(z_{k}(x)\right) \geq \lambda$ ), the system (62) can be written as

$$
\begin{aligned}
& \max \lambda \\
& \text { s.t. } \\
& \mu_{k}\left(z_{k}(x)\right) \geq \lambda, k=1,2, \ldots, K \\
& x \in S \\
& \lambda \in[0,1]
\end{aligned}
$$

Thus, the MOLP problem is converted into the traditional LP problem. The result of (63) is the minimum operator model corresponding to the MOLPP. The optimal value $\left(\lambda^{*}\right)$ corresponds to the value at which the lowest satisfaction level of all objectives is maximized. In other words, it can be interpreted as the most basic satisfaction level of the goals in the given original problem.

\section{A case study}

In this section, the proposed MOMILP model is illustrated in a case study. We study a supply chain network of a company which produces and markets dairy products and plan to implement SCM most efficiently by focusing on customer satisfaction. The network will be managed as effectively as possible by implementing inventory management, which is critical for products with high production and logistical costs in the timeperiod. In the network, plants buy raw material from external suppliers in the form of component/part, assemble recycled parts from recycling centres, and produce finished products under capacity constraints. In this case study, recyclable materials are only packing parts. Moreover, product stock is kept in warehouses to use in the next period when necessary. Customer demand is met from either newly manufactured items, remanufacturing used items collected from customers for recovery, the products that are kept in stock in the warehouse, or from all. Recyclers provide to obtain the returned product from customers, recycle these products to parts, and distribute recycled parts for production. Non-recyclable parts are disposed of with green precautions in the disposal centre. 
The MOCLSC network consists of three external suppliers $(s)$, two candidate plants $(p)$, five candidate warehouses $(w)$, six candidate distribution centres $(d)$, ten customer zones (c), two candidate recycling centres (r), one disposal centre $(k)$. Also, it is supposed that there are four types of products $(j)$ and five types of components $(i)$ with different utilization factor. Moreover, decisions are taken in three time-periods $(t)$. Possible plants, candidate warehouse locations, candidate distribution centre locations, customer zones, candidate recycling centre locations, and disposal centre location in the company's SC network, shown on the representative map (Fig. 2), will provide a better understanding of the results of the proposed model.

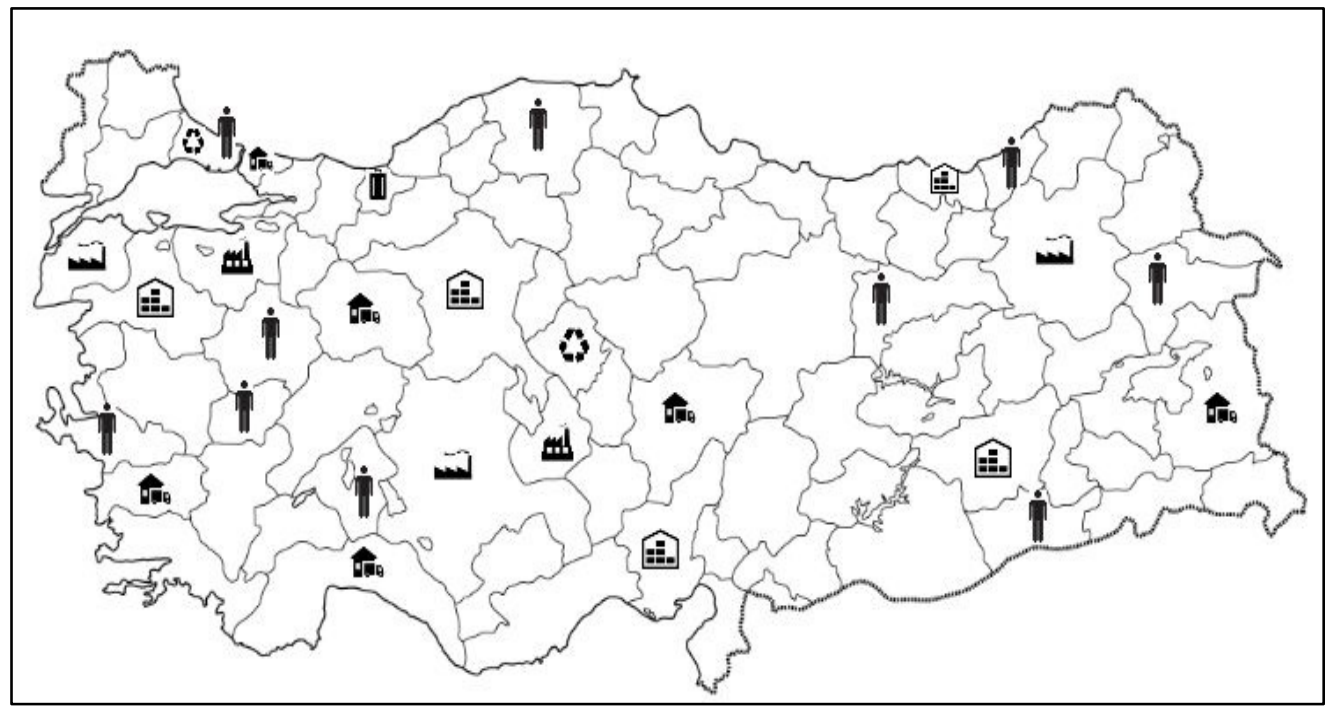

Fig. 2. Company's candidate locations for SC network (including all candidate plant and facilities locations)

Numerical values of some parameters are $L B i=200, L B j=110, \eta_{j}=0.5 \forall j, \lambda_{1}=\lambda_{2}=0$, $\lambda_{3}=0.7, \lambda_{4}=0.6, \lambda_{5}=0.8$, Capikt $_{i k t}=100000000 \forall i, k, t$. We have omitted our enormous data owing to the space limitation of our paper. The results are obtained with the help of the GAMS 23.5 program in $0.047 \mathrm{~s}$. The linear membership functions of objectives are determined as follows to implement the fuzzy approach. These values are given in Table 1.

Table 1. Individual maximum and minimum values of objectives

\begin{tabular}{|c|c|c|}
\hline Objective & Maximum & Minimum \\
\hline 1 & $4.5767 \times 10^{8}$ & $3.2871 \times 10^{7}$ \\
\hline 2 & $4.9699 \times 10^{8}$ & $4.9409 \times 10^{7}$ \\
\hline 3 & $6.8862 \times 10^{8}$ & $5.4077 \times 10^{7}$ \\
\hline
\end{tabular}


By using (60), (61), and the limit values in Table 1, membership functions of objectives are constituted in the interval $\left[Z_{k}^{-}, Z_{k}^{+}\right](k=1,2,3)$. Accordingly, the min operator model obtained is as follows:

$\max \lambda$

s.t.

$$
\begin{aligned}
& \frac{457670000-Z_{1}}{457670000-32871000} \geq \lambda \\
& \frac{Z_{2}-49409000}{496990000-49409000} \geq \lambda
\end{aligned}
$$

$$
\begin{aligned}
& \frac{688620000-Z_{3}}{688620000-54077000} \geq \lambda \\
& x \in X_{T} \\
& \text { where } 0 \leq \lambda \leq 1
\end{aligned}
$$

To be able to interpret and compare the effectiveness of the results, the objectives have been individually solved, and networks have been constituted before solving the problem with the proposed approach. Thus, we got $\lambda=0.702$ with the solution of the model (14). This means that even the least satisfying goal has been observed to be satisfied with $70.2 \%$. The objective functions values and the basic satisfaction level of objectives are given in Table 2.

Table 2. Solution values for objectives

\begin{tabular}{|c|c|c|}
\hline Objective & $z_{k}$ & $\mu_{k}$ \\
\hline 1 & $1.4931 \times 10^{8}$ & 0.726 \\
\hline 2 & $3.6374 \times 10^{8}$ & 0.702 \\
\hline 3 & $2.4299 \times 10^{8}$ & 0.702 \\
\hline
\end{tabular}

Figures 3-5 demonstrate the network created with the results that maximize the common satisfaction of all objectives. The network has emerged that it allows satisfaction of the three objectives at the highest possible level simultaneously. In the optimal solution, when the locations of distribution centres are examined, the facilities are close to the customers who are serviced. This data indicates the consistency in the network. 


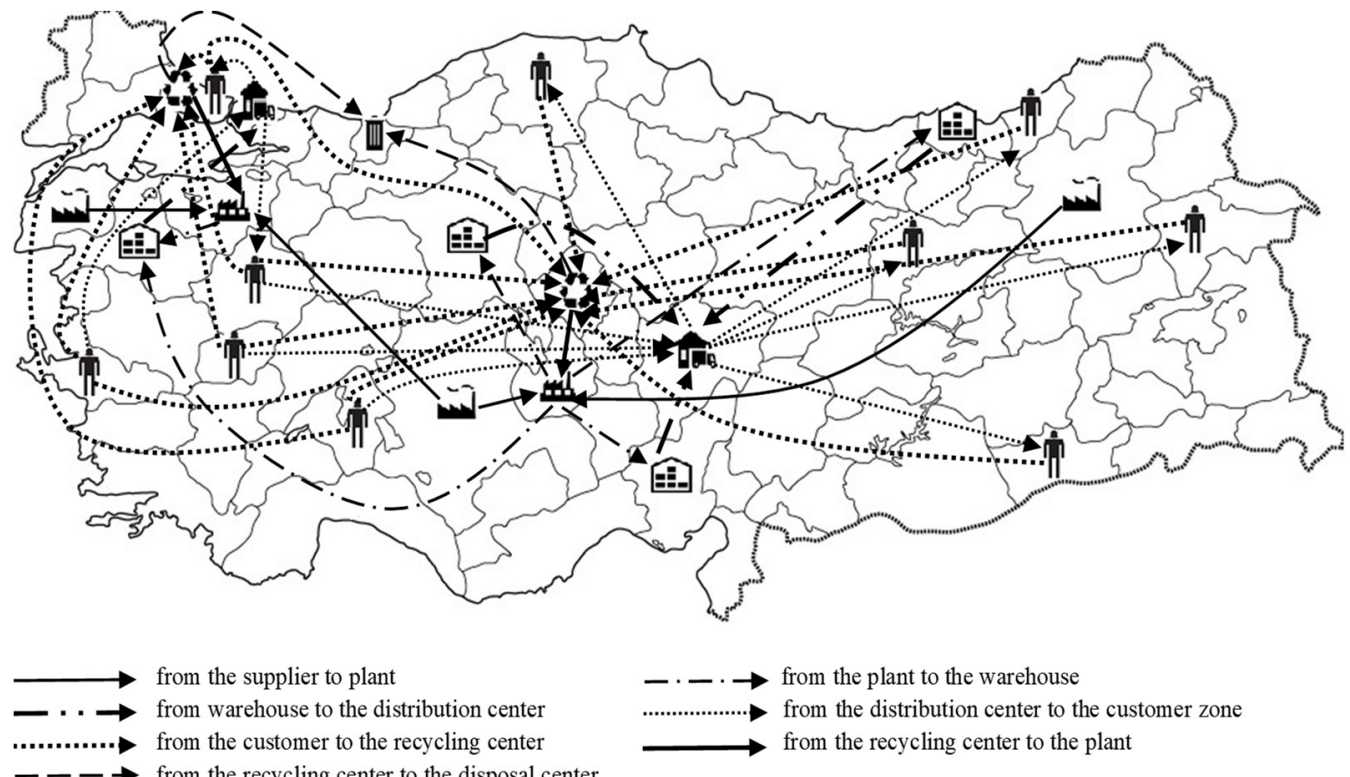

Fig. 3. Company's CLSC network obtained with the maximization of the common satisfaction of all objective functions in the first period

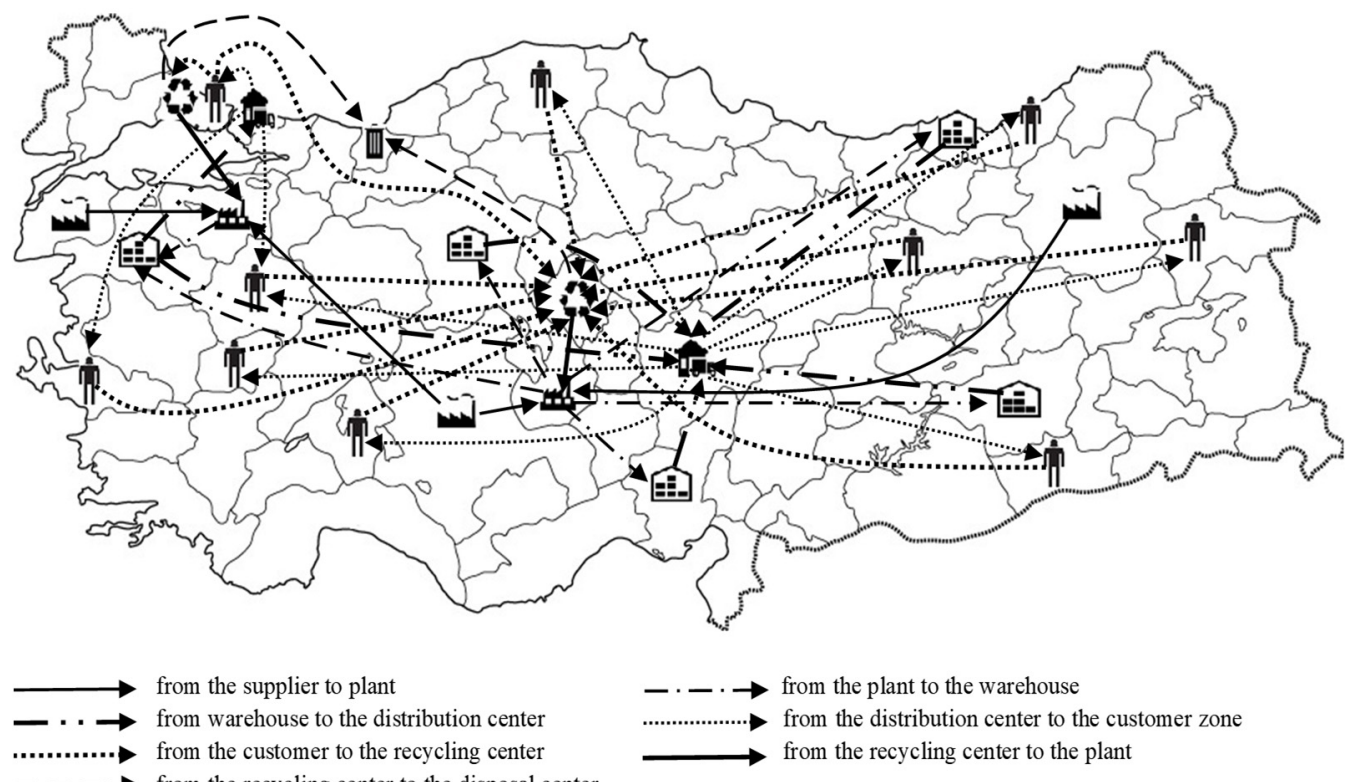

Fig. 4. Company's CLSC network obtained with the maximization of the common satisfaction of all objective functions in the second period 


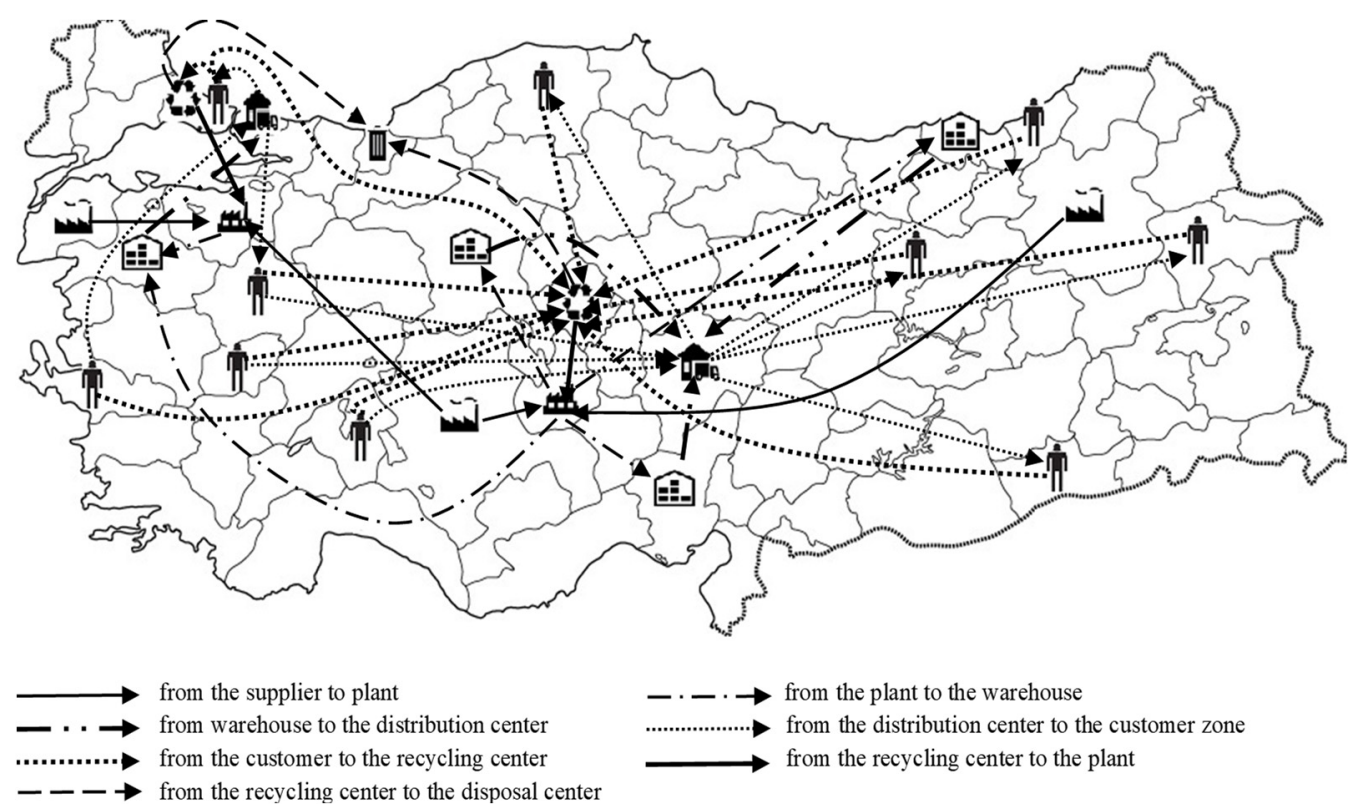

Fig. 5. Company's CLSC network obtained with the maximization of the common satisfaction of all objective functions in the third period

\section{Conclusions}

A multi-echelon CLSC network was designed in which the conflicting objectives were met by mutual compromise at the acceptable satisfaction level. The proposed model was dealt with multi-component, multi-product, multi-plant, multi-warehouses, multi-distribution centres, multi-recycling centres, multi-disposal centres, and multitime-periods. Taking into consideration the $\mathrm{CO}_{2}$ emissions generated from transportation and during the operations in the facilities, the carbon footprint was reduced to the minimum with an environmentalist perspective. Moreover, it was tried to get attention to the importance and necessity of which the addition of recyclable raw materials or semi-products in the reproduction process, the destruction of non-recyclable materials by green methods. Inventory management was also provided throughout the chain to ensure that the production can proceed without hindrance when it was paid regard to periodically increases in customer demands and production costs, and duration of supply due to seasonally diminishing raw materials. The model established in the study was solved in the computer environment with the suggested approach by using GAMS 23.5. The proposed solution methodology was able to generate a balanced solution for the degree of satisfaction between objectives. We achieved a realistic solution by using a fuzzy approach to the solution of our multi-objective model. 
It is considered to work on different approaches fuzzy decision variables and/or parameters to express the uncertainty inherent to the decision-making process for future researches. The model can be adopted under different conditions due to its flexibility. Another future research can be the usage of nonlinear membership functions for this problem.

\section{References}

[1] ILGIN M.A., GUPTA S.M., Environmentally conscious manufacturing and product recovery (ECMPRO). A review of the state of the art, J. Environ. Manage., 2010, 91 (3), 563-591.

[2] Pishvaee M.S., RAZMI J., Environmental supply chain network design using multi-objective fuzzy mathematical programming, Appl. Math. Model., 2012, 36 (8), 3433-3446.

[3] Bowersox D.J., LA Londe B.J., SMYKay E.W., Readings in physical distribution management. The logistics of marketing, Macmillan, 1969.

[4] TSiakis P., Shah N., PANTElides C.C., Design of multi-echelon supply chain networks under demand uncertainty, Ind. Eng. Chem. Res., 2001, 40 (16), 3585-3604.

[5] Melo M.T., Nickel S., Saldanha-DA-Gama F., Facility location and supply chain management. A review, Eur. J. Oper. Res., 2009, 196 (2), 401-412.

[6] Mousavi S.M., Bahreininejad A., Musa S.N., Yusof F., A modified particle swarm optimization for solving the integrated location and inventory control problems in a two-echelon supply chain network, J. Int. Manuf., 2017, 28 (1), 191-206.

[7] Hu T.L., Sheu J.B., HuAng K.H., A reverse logistics cost minimization model for the treatment of hazardous wastes, Transp. Res. Part E: Log. Transp. Rev., 2002, 38 (6), 457-473.

[8] Savaskan R.C., BhatTacharya S., VAN WASSEnHOve L.N., Closed-loop supply chain models with product remanufacturing, Manage. Sci., 2004, 50 (2), 239-252.

[9] Kim K., Song I., KIM J., JEONG B., Supply planning model for remanufacturing system in reverse logistics environment, Comp. Ind. Eng., 2006, 51 (2), 279-287.

[10] AbDallah T., Diabat A., Simchi-Levi D., Sustainable supply chain design: a closed-loop formulation and sensitivity analysis, Prod. Plan. Control, 2012, 23 (2-3), 120-133.

[11] OzCEYlan E., PAKSOY T., A mixed-integer programming model for a closed-loop supply-chain network, Int. J. Prod. Res., 2013, 51 (3), 718-734.

[12] Hasanov P., Jaber M.Y., TAhiROv N., Four-level closed loop supply chain with remanufacturing, Appl. Math. Model., 2019, 66, 141-155.

[13] CHEN C.L., LEE W.C., Multi-objective optimization of multi-echelon supply chain networks with uncertain product demands and prices, Comp. Chem. Eng., 2004, 28 (6-7), 1131-1144.

[14] Peidro D., Mula J., Poler R., Verdegay J.L., Fuzzy optimization for supply chain planning under supply, demand and process uncertainties, Fuzzy Sets Syst., 2009, 160 (18), 2640-2657.

[15] OZKOK B.A., TIRYAKI F., A compensatory fuzzy approach to multi-objective linear supplier selection problem with multiple-item, Exp. Syst. Appl., 2011, 38 (9), 11363-11368.

[16] Shi J., Zhang G., SHa J., Optimal production planning for a multi-product closed loop system with uncertain demand and return, Comp. Oper. Res., 2011, 38 (3), 641-650.

[17] Amin S.H., ZHANG G., An integrated model for closed-loop supply chain configuration and supplier selection: Multi-objective approach, Exp. Syst. Appl., 2012, 39 (8), 6782-6791.

[18] Ramezani M., Kimiagari A.M., Karimi B., Hejazi T.H., Closed-loop supply chain network design under a fuzzy environment, Knowl.-Based Syst., 2014, 59, 108-120. 
[19] Jindal A., Sangwan K.S., Closed loop supply chain network design and optimisation using fuzzy mixed-integer linear programming model, Int. J. Prod. Res., 2014, 52 (14), 4156-4173.

[20] Jindal A., SANGWAN K.S., SAXENA S., Network design and optimization for multi-product, multi-time, multi-echelon closed-loop supply chain under uncertainty, Proc. CIRP, 2015, 29, 656-661.

[21] Soleimani H., Govindan K., Saghafi H., Jafari H., Fuzzy multi-objective sustainable and green closed-loop supply chain network design, Comp. Ind. Eng., 2017, 109, 191-203.

[22] Chen Y.T., ChAn F.T., ChUnG S.H., PARK W.Y., Optimization of product refurbishment in closed-loop supply chain using multi-period model integrated with fuzzy controller under uncertainties, Rob. Comp.-Int. Manuf., 2018, 50, 1-12.

[23] Wu G.H., Chang C.K., Hsu L.M., Comparisons of interactive fuzzy programming approaches for closed-loop supply chain network design under uncertainty, Comp. Ind. Eng., 2018, 125, 500-513.

[24] ZimMERMANN H.J., Fuzzy programming and linear programming with several objective functions, Fuzzy Sets Syst., 1978, 1 (1), 45-55. 